\title{
The use of proper orthogonal decomposition for the simulation of highly nonlinear hygrothermal performance
}

\author{
Tianfeng Hou ${ }^{1 *}$, Staf Roels ${ }^{1}$ and Hans Janssen ${ }^{1}$ \\ ${ }^{1}$ KU Leuven, Department of Civil Engineering, Building Physics Section, 3001 Leuven, Belgium
}

\begin{abstract}
In this paper, the use of proper orthogonal decomposition for simulating nonlinear heat, air and moisture transfer is investigated via two applications: HAMSTAD benchmarks 2 and 3. Moreover, the potential of the reduced models constructed by proper orthogonal decomposition for simulating new problems with longer simulation periods is assessed. To illustrate the feasibilities of proper orthogonal decomposition method in the field of building physics, the accuracies of the reduced models are compared with the standard finite element method. The outcomes show that with a sufficient number of construction modes and a relatively large amount of snapshots, proper orthogonal decomposition method can deliver accurate results. In addition, guidelines on selecting an appropriate amount of simulation snapshot and construction modes are provided.
\end{abstract}

\section{Introduction}

The use of numerical simulations for hygrothermal performance assessment is becoming progressively more common, given that a multitude of scenarios and factors can be evaluated in such numerical analysis. However, the conventional numerical methods based on the space and time discretization can be very time consuming due to the high nonlinearity of the equations, the complex multi-dimensional spatial domains and the long time intervals required [1]. Therefore, a faster surrogate model is highly desired. Instead of using the conventional numerical models, Van Gelder et al [2] employed metamodels derived by regression and interpolation (polynomial regression, Kriging, etc.) to reduce the simulation time. However, these simpler surrogate models can only deliver static results: for heat and moisture transfer through a building component, they may predict the yearly total heat loss or yearly average moisture content, but not the evolution of temperature or moisture content profiles over time. Hence, to obtain the dynamic behavior with a faster surrogate model, in this paper one common model order reduction technique - proper orthogonal decomposition (POD) - is investigated as alternative of the statistical surrogate modelling.

The key idea of POD is approximating a high-dimensional process by its 'most relevant information', and as a result the number of degrees of freedom of the system can commonly be strongly reduced without losing the dynamic behavior of the original model. To illustrate

* Corresponding author: tianfeng.hou@kuleuven.be 
the performance of POD in the field of building physics, the accuracy of using POD for simulating nonlinear hygrothermal performance and the robustness of using the constructed reduced POD models for simulating new problems with longer simulation time periods are evaluated via two applications: HAMSTAD benchmarks 2 and 3 [3]. The outcomes show that with a sufficient number of construction modes and a relatively large amount of snapshots, the POD method can deliver accurate results and is able to predict the results of new problems with longer simulation time periods.

Below, first a brief introduction of POD is put forward, with focus on the potential use for the simulation of nonlinear heat, air and moisture transfer. Subsequently, two illustrative case studies: HAMSTAD benchmarks 2 and 3 are introduced, being the central applications in this study. Next, the results of using POD for simulating nonlinear heat, air and moisture transfer are presented and a discussion with respect to the interpretation of its accuracy and robustness follows. Finally, conclusions on the accuracy of POD and the capability of POD for predicting new problems are formulated, together with a guideline about the selection of an appropriate number of simulation snapshots and construction modes.

\section{POD for the simulation of building performance}

The hygrothermal performance of a building component can be assessed by analyzing the transfers of heat, air and moisture through building materials, requiring to get numerical simulation results for the coupled heat, air and moisture transfer equations with information on component geometry, material properties and boundary conditions. However, for highly nonlinear hygrothermal simulations, traditional approaches such as the finite element method (FEM), can be very time consuming, the main reason being that in each of the iterations, we need to solve a large system of equations due to the high number of degrees of freedom after the spatial discretization. Normally, the stronger the nonlinearities we have in building materials and/or boundary conditions, the finer the spatial and temporal discretization grids should be, and these factors can strongly increase the number of degrees of freedom of our simulation system. Therefore, in this paper we investigate the POD method which has the potential to reduce this number of degrees of freedom of the complex system while still mimicking the dynamic behavior (such as e.g. the time evolution of temperatures, moisture content...).

The POD method was first proposed by Kosambi [4], and has been successfully applied in a variety of engineering fields, such as image processing, signal analysis, data compression and recently in building physical engineering [1, 5-7]. However, to the best of the authors' knowledge, using POD to simulate nonlinear hygrothermal performance in the field of building physics is rarely mentioned in the literature. And research questions about how to select an appropriate number of simulation snapshots and construction modes in order to build the basis for an accurate surrogate model remain unclear. Therefore, this paper studies the use of POD for dealing with nonlinear hygrothermal simulation, with emphasis on the selection of snapshots and modes. In other words, as the key idea of POD is approximating a high-dimensional process by its 'most relevant information', this paper aims at providing a guideline about using the smallest number of snapshots and modes to retain the most of the crucial information. In this paper we extract that 'most relevant information' from the simulation snapshots with a singular value decomposition (SVD). After the SVD, the POD modes are constructed by selecting a basis with the $\mathrm{k}$ most important modes, here $\mathrm{k} \ll \mathrm{N}$, where $\mathrm{N}$ is the number of the original spatial mesh elements. As a result, these POD modes can be used to construct a reduced model for simulating new problems (for instance, variations in the boundary conditions, variations in the material properties, variations in the component geometry, or longer simulation period). For detailed procedures on using POD to construct the reduced-order-model, we refer the readers to [8]. 


\section{Case studies}

For investigating the potential use of POD for hygrothermal simulations, a reference benchmark which can assess the performance of different simulation models is required. In this paper, two applications, HAMSTAD benchmarks 2 and 3 [3], are selected as the case studies. Both have a one-dimensional geometrical structure, but are based on different transfer mechanisms with different combinations of boundary conditions and material properties. More specifically, HAMSTAD benchmark 2 analyzes the isothermal moisture redistribution in one material layer caused by sudden drops of the relative humidity of the surroundings. On the other hand, HAMSTAD benchmark 3 centers on air transfer through a light weight wall, wherein the moisture and heat transfer are mainly driven by the air flow. Detailed information about these two case studies and their related construction geometries, material properties and boundary conditions can be found in [3].

In order to evaluate the performance of POD as a function of the number of construction modes, for both of the case studies the results derived by the standard FEM and the reduced models constructed by different number of modes ( 30 modes, 10 modes, 5 modes, 1 mode) are compared. The simulation results of the FEM are taken here as reference solution. More specifically, for HAMSTAD benchmark 2 and 3 the reference solutions are respectively calculated by the FEM with 200 and 40 spatial mesh elements. In addition, as mentioned in section 2, as POD is constructed for simulating new problems, for HAMSTAD benchmark 3 , several POD models are constructed by using snapshots from different time intervals ( 50 days, 30 days, 20 days, 10 days, 5 days and 1 day), and these reduced models are used to predict the results of a new problem with a time period of 100 days.

\section{Results}

In this section, the performance of POD is evaluated via two case studies: HAMSTAD benchmarks 2 and 3 . The respective results are presented in the two subsections below.

\subsection{HAMSTAD benchmark 2}

For getting a direct view of the performance of POD in relation to HAMSTAD benchmark 2 , the moisture content profiles at 100 hours of the reduced models constructed by different number of modes together with the results derived by the reference solution are shown in Figure 1.

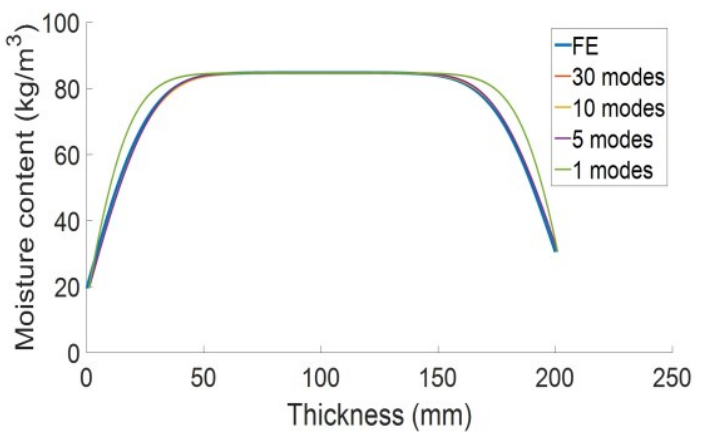

Fig. 1. Moisture content profiles at 100 hours of the FE solution and solutions of the reduced models constructed by different number of modes. 
Figure 1 shows that the differences between the reference solution and the results of the other POD models are very small, except for the POD model constructed by 1 mode. With more than 10 construction modes, there is no observable differences between the reference solution and the POD approximations. Hence, for HAMSTAD benchmark 2, with a relatively small number of construction modes, the POD method can provide an accurate result of the moisture distribution in the material layer.

\subsection{HAMSTAD benchmark 3}

In the case study of HAMSTAD benchmark 3, two research questions are studied: 1) the accuracy of POD as a function of the amount of construction modes when simulating the same problem, and 2) the accuracy of POD as a function of the size of snapshot and the amount of construction modes when simulating new problems with longer simulation periods.

\subsubsection{The performance of $P O D$ when simulating the same problem}

In relation to HAMSTAD benchmark 3, both temperature and moisture distributions at 0.19 $\mathrm{m}$ in the building component simulated by different reduced models together with the result of the reference solution are shown in Figure 2.
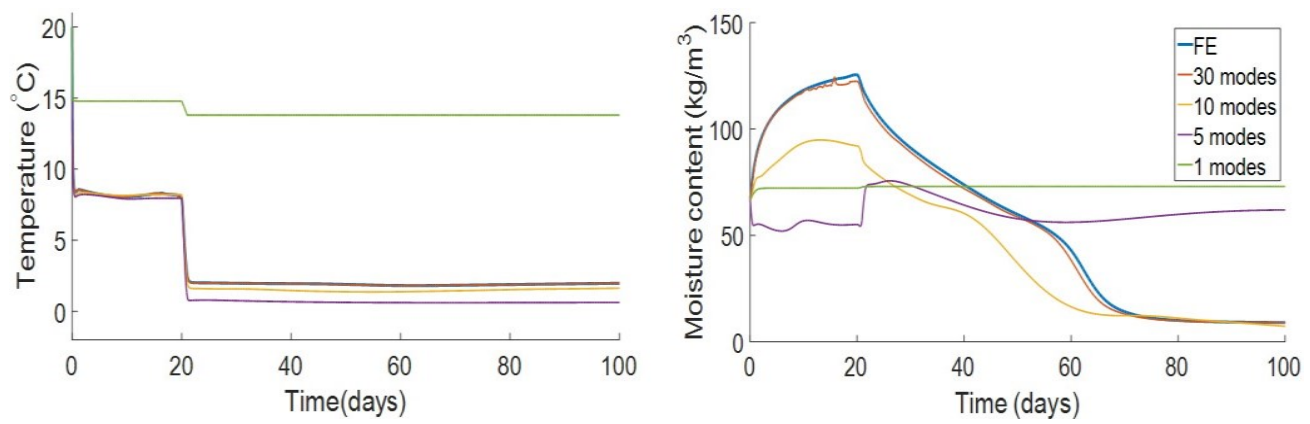

Fig. 2. Temperature and moisture content distributions at $0.19 \mathrm{~m}$ of the building component simulated by the FEM and the reduced models constructed by different number of modes.

Figure 2 illustrates the temperature and moisture distributions at $0.19 \mathrm{~m}$ of the building component simulated by different models. It is demonstrated that, the accuracy of POD method increases as the number of its construction modes raises. With respect to the temperature distributions, we notice that with 10 construction modes, the distribution of the temperature can be quantified rather accurately. On the other hand, it is shown that for obtaining a relatively accurate distribution of the moisture content, at least 30 modes are needed. Therefore, comparing with quantifying the temperature distribution, due to the stronger nonlinear behaviour of the moisture transfer mechanism, obtaining the moisture content profiles requires more construction modes. 


\subsubsection{The performance of $P O D$ when simulating new problems with longer periods}

Before, it is shown that the POD model constructed based on the full set of snapshots can provide an accurate result when simulating the same system. However, in practice this recalculation of the same problem does not add any value, as all the information is already included in the matrix of snapshots. Instead, in this section, we are investigating the use of POD to construct a reduced model for simulating different problems in relation to longer simulation period. In other words, the accuracies of the POD models constructed by different size of snapshots are evaluated. More specifically, for getting a more direct view of the performance POD method as a function of the size of its snapshot, similar as before both temperature and moisture distributions at $0.19 \mathrm{~m}$ in the building component simulated by FEM and the reduced models constructed by 25 modes and different size of snapshots are shown in Figure 3. In addition, in order to also evaluate the accuracy of POD method as a function of the amount of the construction modes, the average temperature and moisture content differences between the reference solution and POD models built by different size of snapshots, as a function of the amount of their construction modes are shown in Figure 4.
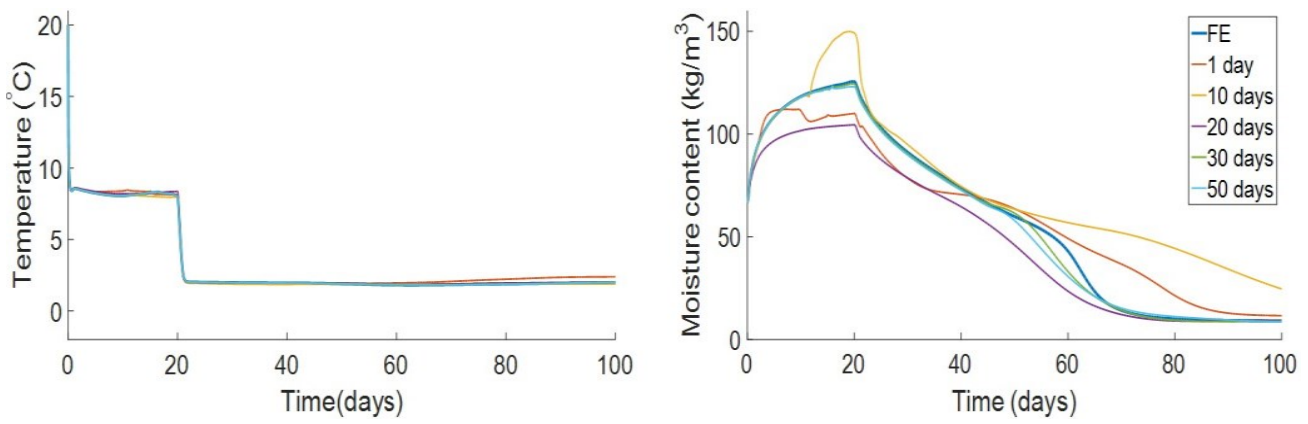

Fig. 3. Temperature and moisture content distributions at $0.19 \mathrm{~m}$ of the building component simulated by the FEM and reduced models constructed by 25 modes and different size of snapshots.

Figure 3 is similar to Figure 2, but now for different size of snapshots. It is demonstrated that, with only one day of snapshot and 25 modes, the temperature distribution can be obtained rather accurately. On the other hand, relative to the temperature distribution, due to the stronger nonlinear behaviour of the moisture transfer mechanism, obtaining the moisture content profiles requires a much larger size of snapshot.
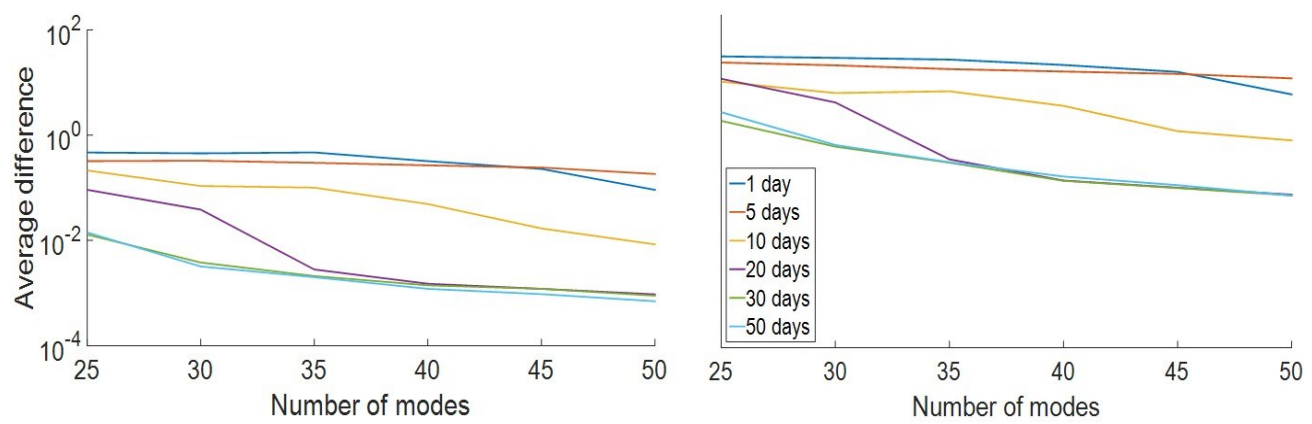

Fig. 4. Average temperature and moisture content differences between the FE solution and solutions of the reduced models constructed by different size of snapshots and different number of modes. 
Figure 4 confirms the results of Figure 2 and Figure 3, the accuracy of POD method increases as the amount of their construction modes raises and as the size of their snapshot grows. Comparing with the average temperature differences between the reference solution and the results of different POD models, the average moisture content differences are a lot larger (larger than one magnitude). Hence with the same size of snapshot, quantifying the distribution of the moisture content requires more construction modes than capturing the distribution of the temperature. In addition, Figure 4 demonstrates that, with less than 30 modes, a relatively large accuracy differences are found between the results of the reduced models constructed by the snapshots of 20 days and 30 days. One possible reason is that for HAMSTAD benchmark 3, since there is a large change of the air pressure gradient from the 20 days to the 21 days [3], the snapshots include this period contain the crucial information of the boundary condition and hence has the potential to be the base of a more accurate reduced model.

\section{Discussion}

When combining all the findings, we conclude that the use of POD for simulating nonlinear hygrothermal performance has the potential to provide an accurate result and is able to predict the results of a new problem with longer simulation period. However, caution should be taken when we choose the number of simulation snapshots and construction modes to build a reduced model. In relation to the snapshots, it is strongly recommended to include the crucial information and the periodic patterns of the boundary conditions as much as possible. On the other hand, with respect to the modes, choosing an appropriate number of modes depends on the nonlinearity of the target problem. Normally the stronger the nonlinearity we have, the more modes we need in order to obtain an accurate result. Therefore, in practice we could first select an appropriate size of snapshot and consider it as the reference solution. Next, we constructed the reduced model by keeping adding modes and comparing the result with the reference solution until no further improvement can be obtained. Finally, we could use the constructed reduced model for simulating new problems with a longer simulation time period.

This work has been supported by the H2020 RIBuild project, their support is gratefully acknowledged.

\section{References}

1. T. Hou, G. Roels, H. Janssen, In Proceedings IBPC 2018, 1295-1300 (2018).

2. L. Van Gelder, P. Das, H. Janssen, S. Roels, SIMUL MODEL PRACT TH, 49, 245257 (2014).

3. C.E. Hagentoft, HAMSTAD-Final report, 19-23 (2002).

4. D.D. Kosambi, D.D. Kosambi: selected works in mathematics and statistics (Springer, 115-123, 2016).

5. A. Tallet, C. Allery, F. Allard, BUILD ENVIRON 93, 34-49 (2015).

6. A. Tallet, E. Liberge, C. Inard, BUILD SIMUL-CHINA 10, 111-121 (2017).

7. J. Berger, W. Mazuroski, R.C. Oliveira, N. Mendes, J BUILD PERFORM SIMU 11, 120 (2018).

8. M. Agudelo, The application of proper orthogonal decomposition to the control of tubular reactors, $\mathrm{PhD}$ thesis (2009). 